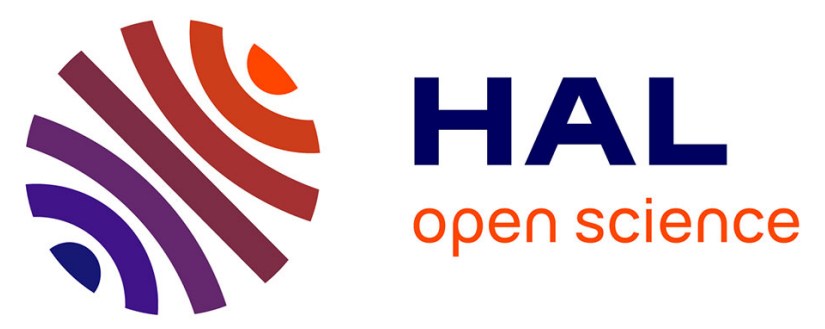

\title{
Increase of precuneus metabolism correlates with reduction of PTSD symptoms after EMDR therapy in military veterans: an 18 F-FDG PET study during virtual reality exposure to war
}

P. Philippe Rousseau, E. Malbos, A. Verger, F. Nicolas, C. Lançon, S. Khalfa, Eric Guedj

\section{To cite this version:}

P. Philippe Rousseau, E. Malbos, A. Verger, F. Nicolas, C. Lançon, et al.. Increase of precuneus metabolism correlates with reduction of PTSD symptoms after EMDR therapy in military veterans: an 18F-FDG PET study during virtual reality exposure to war. European Journal of Nuclear Medicine and Molecular Imaging, 2019, 46 (9), pp.1817-1821. 10.1007/s00259-019-04360-1 . hal-02479693

\section{HAL Id: hal-02479693 \\ https://hal.science/hal-02479693}

Submitted on 6 Apr 2020

HAL is a multi-disciplinary open access archive for the deposit and dissemination of scientific research documents, whether they are published or not. The documents may come from teaching and research institutions in France or abroad, or from public or private research centers.
L'archive ouverte pluridisciplinaire HAL, est destinée au dépôt et à la diffusion de documents scientifiques de niveau recherche, publiés ou non, émanant des établissements d'enseignement et de recherche français ou étrangers, des laboratoires publics ou privés. 


\section{Responses to Reviewers}

Reviewers' comments:

Reviewer \#1: Comments to Editor

The manuscript "Increase of precuneus metabolism correlates with reduction of PTSD symptoms after EMDR therapy in military veterans: an 18F-FDG PET study during virtual reality exposure to war" by Rousseau et al deals with very actual topic: the disclosure of the neurobiology of psychotherapies. Furthermore, approaching such matter by PET activation studies, after about 30 years since the first 15O-H2O ones, using FDG might start a new season for this fascinating research field

The manuscript is concise, well written and pretty clear in the aims, methods, results and discussion and in my opinion misses only a few information that will would improve its quality.

I have a general comment and some minor specific remarks

General comments

The authors have recently published on the EJNMMI an editorial promoting functional 18FFDG PET brain activation imaging. A similar study to the present one was published on the same Journal in 2015 (Eur J Nucl Med Mol Imaging (2015) 42:733-740) in which the very same protocol and methodology were used with the only difference that the stressing stimulus was olfactory and not visual.

Since the readers of the present paper will be (firstly) nuclear medicine physicians it is opinion of this reviewer that the above paper should be quoted and the methodology implemented in both of them better "promoted" in the introduction and discussion to stimulate the colleagues to perform similar studies.

It is also the opinion of this reviewer that it is a pity that the authors decided to submit such interesting and ground-breaking paper as short communication instead of full paper.

Reply: Thanks to the Reviewer for his/her highly positive comments. As mentioned, we are limited by the constraints of a short communication that we indeed chose considering this work as a pilot study with the inclusion of only fifteen subjects. At the discretion of the Editor, the paper could possibly be considered for an Editorial to highlight the original points raised by the Reviewer. Nevertheless, the text has been reasonably lengthened on this revision to better include these aspects. In details, the use of ${ }^{18} \mathrm{~F}$-FDG brain PET imaging for activation studies has now been promoted in regard to our recent published articles (ref 5 and 7), but also the interesting recommended reference proposed by the Reviewer (ref 6), in the Introduction (p3, 119-22): "Indeed, the use of ${ }^{18} F-F D G$ brain PET imaging for activation studies has been recently proposed to investigate brain metabolic changes during stimuli tasks such as olfactory stimulations or VRE tasks.", and in the Discussion (p7, 16-12): "Therefore, this study highlights the exciting opportunity to use PET imaging to investigate 
brain metabolism during virtual exposure, and provides additional information on the pathophysiological response of EMDR therapy on PTSD. In comparison to functional magnetic resonance imaging (fMRI), the competitive advantage of ${ }^{18} F-F D G$ brain PET imaging is to possibly capture an activation paradigm outside the acquisition scanner, during the radiopharmaceutical injection, and thus possibly develop complex paradigms involving for example VRE. In this line, this approach could be extended to other virtual environments or neuropsychological conditions."

\section{Minor remarks}

In the Introduction line 19-20 it should be made clear that the study quoted in reference 4 was performed by EEG, not fMRI.

Reply: This has been modified accordingly in the Introduction (p3, 112-13): "Previous studies showed functional changes in structures involved in the fear network using fMRI (3) and EEG (4) on activation tasks after EMDR."

At page 5, first paragraph the authors should be more precise about the chosen statistical threshold ( $p$ valure corrected/not corrected for multiple comparisons; in case FDR o FWE corrected?). The choice of cluster volume is not a correction, is a size threshold.

Reply: Thanks for this remark. The statistical threshold was $\mathrm{p}<0.005$ uncorrected, with a $\mathrm{k}$ size threshold $>180$. This choice has been made to limit type-II errors.

It has been modified accordingly throughout the manuscript.

At page 7, lines 22-25 the authors speculate about precuneus and DMN. I would not go into such discussion since the DMN is active at rest and in the present case its increased metabolism is found during emotional activation.

Reply: We agree with the Reviewer. We have modified this sentence by deleting the term DMN in the Discussion (p7, 121-24): "Due to its functions, we can assume that the precuneus can modulate the subjective experience of anxious and fearful states to reprocess the traumatic memory and discharge it from its emotional load".

Reviewer \#2: Straightforward report of small study of PTSD patients scanned with FDG-PET before and after EMDR treatment. Clearly described and interpreted with due care and caution regarding the small sample.

Reply: Thanks to the Reviewer for his/her positive comments. 


\section{Increase of precuneus metabolism correlates with reduction of PTSD symptoms after EMDR therapy in military veterans: an 18F-FDG PET study during virtual reality exposure to war}

Rousseau $\mathrm{PF}^{1}$, Malbos $\mathrm{E}^{2}$, Verger $\mathrm{A}^{3,4}$, Nicolas $\mathrm{F}^{5}$, Lançon $\mathrm{C}^{2}$, Khalfa $\mathrm{S}^{1}$, Guedj $\mathrm{E}^{6,7,8}$

1: Laboratoire de Neurosciences Sensorielles et Cognitives, Aix-Marseille Université CNRS, Marseille, France

2: Department of Psychiatry, La Conception University Hospital, Marseille, France

3: Department of Nuclear Medicine \& Nancyclotep Imaging platform, CHRU Nancy, Lorraine University, France

4: IADI, INSERM, UMR 1254, Lorraine University, Nancy, France

5: Service de Psychiatrie, Hôpital d'Instruction des Armées Sainte-Anne, Toulon, France

6: Aix-Marseille Université, CNRS, Ecole Centrale de Marseille, UMR 7249, Institut Fresnel, Marseille, France

7: Department of Nuclear Medicine, Assistance Publique Hôpitaux de Marseille, Timone University Hospital, France

8: CERIMED, Aix-Marseille University, Marseille, France

Running title: PET metabolism after EMDR in PTSD

Word count: 1,832

\section{Address for correspondence:}

Eric Guedj, MD, PhD

eric.guedj@ap-hm.fr

Service Central de Biophysique et Médecine Nucléaire, Hôpital de la Timone, 264 rue Saint Pierre, 13005 Marseille, France.

Tel: +33-491385558; Fax: +33-491384769 


\begin{abstract}
Purpose: The prevalence of posttraumatic stress disorder (PTSD) is higher among veterans and can lead to disastrous consequences such as suicide. Eye Movement Desensitization and Reprocessing (EMDR) is recommended in first-line psychotherapies for PTSD. Virtual reality exposure (VRE) coupled with 18F-FDG PET imaging can highlight the activated brain regions during stress exposure. The objective of this study is to identify, after EMDR therapy, the regions of brain metabolism that evolve during the stress exposure of a war scene with symptomatic remission in a group of military veterans suffering from PTSD and to secondarily search for predictive metabolic features.
\end{abstract}

Methods: We recruited fifteen military veterans suffering from PTSD who performed an ${ }^{18} \mathrm{~F}$ FDG PET sensitized by the exposure to a virtual war scene, before (T0) and after (T1) EMDR therapy. Statistical parametric mapping was used to compare brain metabolism before and after treatment and to study correlations between metabolism and evolution scores on PTSD clinical scales (PTSD Checklist Scale, PCLS; Clinician Administered PTSD Scale, CAPS).

Results: The metabolic activity of the precuneus was increased after EMDR therapy $(p<0.005$ uncorrected, $\mathrm{k}>180)$ and correlated with clinical improvement with the CAPS scale ( $\mathrm{r}=-0.73$ and $\mathrm{p}<0.001)$. Moreover, the precuneus metabolic value before therapy predicted the clinical improvement on the PCLS scale (T1-T0) after EMDR $(r=-0.667$ and $p<0.006)$.

Conclusion: The clinical improvement in military patients with PTSD after EMDR is related to increased precuneus metabolism upon VR stress exposure.

\title{
Keywords
}

Posttraumatic Stress Disorder; Brain Metabolism; PET; Eye Movement Desensitization and Reprocessing; Virtual Reality Exposure; War; Veterans 


\section{Introduction}

Posttraumatic stress disorder (PTSD) is a condition that can develop following an exposure to traumatic events. The rates of PTSD in veterans are higher than in the civilian population, at close to $35 \%$ of Army and Marine troops having PTSD after deployment (1).

The pathophysiology of PTSD has been studied with neuroimaging and paradigms such as script-driven imagery and visual emotional stimuli, demonstrating brain abnormalities in the fear network, in the reward and motivation network, and in the default mode network (2).

Eye Movement Desensitization and Reprocessing (EMDR) therapy is recommended in firstline psychotherapies for PTSD. EMDR includes associations of cognitive, emotional and physical assessments of actual distress to the traumatic scenery during bilateral alternating stimulations (auditory, visual, tactile stimuli alternating between the two sides of the body). Previous studies showed functional changes in structures involved in the fear network using fMRI (3) and EEG (4) on activation tasks after EMDR.

Displaying more ecological environments is now possible with virtual reality exposure (VRE), which allows patients to be immersed in a computer-generated virtual environment mimicking real-life in laboratory conditions. VRE provides the unique opportunity to study pathophysiological changes in an ecologically relevant setting during simulated trauma event exposure. Interestingly, VRE can also be associated with 18F-FDG PET imaging to measure brain metabolism during confrontation with the traumatic event (5). Indeed, the use of ${ }^{18} \mathrm{~F}$-FDG brain PET imaging for activation studies has been recently proposed to investigate brain metabolic changes during stimuli tasks such as olfactory stimulations (6) or VRE tasks (7).

The objective of this study is to identify, after EMDR therapy, the regions of brain metabolism that evolve during stress exposure to a war scene with symptomatic remission in a group of military veterans suffering from PTSD and to secondarily search for predictive metabolic features. 


\section{Materials and Methods}

\section{Participants}

Military PTSD patients were recruited in the military hospital Sainte-Anne at Toulon, France. Diagnosis of PTSD was established according to the DSM-IV TR (8). We excluded patients with present and/or past neurological or psychiatric conditions, with the exception of anxiety and depressive disorders if their occurrence was connected with PTSD. Patients with an addictive disorder, even in relation to PTSD, were excluded. We used the Posttraumatic Stress Checklist Scale (PCLS) (9), which is a brief and self-report questionnaire for evaluating the severity of the three main syndromes of PTSD, and the Clinician-Administered PTSD Scale (CAPS) (10), which is a structured interview providing a categorical diagnosis, as well as a measure of the severity of PTSD symptoms as defined by the DSM.

All the soldiers included had PTSD related to war combat (Afghanistan and Mali). None had received formal exposure or cognitive-behavioural therapy before the EMDR procedure. Sociodemographic characteristics, including comorbidity, medications, and duration of illness, are described in Table 1 .

\section{EMDR procedure}

EMDR therapy was performed according to the standard protocol. Sessions were planned every 7 to 15 days according to the availability of patients and therapists. Patients could receive a maximum of eight EMDR sessions depending on their clinical course. All patients were symptom-free and no longer diagnosed with PTSD after EMDR therapy, as assessed by a psychiatrist diagnosis according to DSM-IV criteria and clinical PTSD scales.

\section{${ }^{18}$ F-FDG-PET Acquisition and Analysis}

${ }^{18}$ F-FDG PET was performed using an integrated PET/CT camera (Discovery 710, GE Healthcare, Waukeskha, WI) with parameters previously described (7). Each subject was confronted with intense trauma cues, consisting of the attack on their group by insurgents during a patrol in an artificially created village in Afghanistan, with several virtual soldiers wounded, (screen captures in Figure 1). Patients were exposed to the environment approximately $10 \mathrm{~min}$ before the injection. ${ }^{18} \mathrm{~F}$-FDG $(150 \mathrm{MBq})$ was injected intravenously one minute before their unit was assaulted, and the virtual exposure immersion was maintained after injection for approximately $7 \mathrm{~min}$. Thereafter, patients were placed in a quiet environment with their eyes closed but continued to feel the stress of the VRE. PET images, acquired in a lying position as recommended in standard practice, started $30 \mathrm{~min}$ after the injection and ended $15 \mathrm{~min}$ later. Finally, patients were asked to indicate the fear felt during the VRE. The degree of fear was measured by an SUD for subjective units of discomfort ranging from 0 (no disturbance) to 10 (maximum disturbance) as previously described (7). 
Whole-brain statistical analysis was performed at the voxel level using SPM8 software (Wellcome Department of Cognitive Neurology, University College, London, UK) as previously reported (7). SPM (T) maps were generated for comparison before and after EMDR therapy using paired t-tests ( $p<0.005$ uncorrected, $k>180$ ). Mean values of metabolism were extracted at the individual level for significant cluster(s) to calculate correlations with clinical scores (PCLS and CAPS scores at T0 (before therapy), T1 (after therapy) and T1-T0).

\section{Statistical analysis}

Spearman correlations were performed between the PCLS and CAPS scores at T0, T1, for T1$\mathrm{T} 0$ and the metabolism of significant cluster(s) extracted from the paired-SPM analysis performed before and after EMDR and their related changes. We applied a Bonferroni correction $(\mathrm{k}=8$ and $\mathrm{p}<0.00625)$. Scores on clinical scales have been compared by paired t-tests. A p-value $<0.05$ was considered significant. 


\section{Results}

\section{Patients and changes in PET metabolism after EMDR therapy}

The EMDR group was composed of seventeen military participants (mean age $36.8 \pm 5.9$ years). Of all patients enrolled, two were excluded from the analyses, one having not completed EMDR therapy and the other not being able to repeat the second PET after therapy. Significant clinical improvements were noticed after EMDR as described in Table 1 for PTSD scales (PCLS, CAPS) and SUD scores ( $p<0.001$ for all scores). The second 18F-FDG PET was performed during the month in which the patient was declared in remission for PTSD.

There was a positive correlation between the evolution of the SUD (T1 - T0) and the PCLS (T1 $-\mathrm{T} 0$ ), $\mathrm{r}=0.74$ and $\mathrm{p}<0.001$. The more disturbances decreased during the VRE, the higher the symptoms improved after EMDR therapy.

The comparison between the patients assessed with ${ }^{18} \mathrm{~F}$-FDG PET before and after EMDR therapy showed an increased metabolism in the bilateral precuneus with a right-predominance after treatment $(\mathrm{p}<0.005$ uncorrected, $\mathrm{k}>180)$, as shown in Figure 2. No decreased metabolism was found.

\section{Relationship between clinical improvement after EMDR and precuneus metabolism}

There was a negative correlation between the difference in metabolic values $(\mathrm{T} 1-\mathrm{T} 0)$ of the precuneus and the evolution of the CAPS (T1 $-\mathrm{T} 0$ ), with $r=-0.73$ and $p<0.001$. The higher the metabolic value of the precuneus increased after EMDR, the more the symptoms improved. There was a negative correlation between the metabolic value in the significant cluster precuneus before EMDR and the evolution of the PCLS (T1 - T0), with $r=-0.667$ and $p<$ 0.006. The higher the precuneus metabolism before EMDR, the more the PCL-S scores decreased at the end of the therapy. The two correlations are depicted in Figure 3.

No other correlation was found between the clinical scores and precuneus metabolism. 


\section{Discussion}

The objective of this study was to identify the regions of brain metabolism that evolve after EMDR therapy with symptomatic remission during stress exposure of a war scene in a group of military veterans suffering from PTSD. One of the most novel aspects is the use of PET imaging combined with VRE sensitization to measure brain metabolism during the interaction with the traumatic event. Therefore, this study highlights the exciting opportunity to use PET imaging to investigate brain metabolism during virtual exposure, and provides additional information on the pathophysiological response of EMDR therapy on PTSD. In comparison to fMRI, the competitive advantage of ${ }^{18} \mathrm{~F}$-FDG brain PET imaging is to possibly capture an activation paradigm outside the acquisition scanner, during the radiopharmaceutical injection, and thus possibly develop complex paradigms involving for example VRE. In this line, this approach could be extended to other virtual environments or neuropsychological conditions.

The SUD evolution correlated with the evolution of the PCLS. This validates that our virtual environment was effective in reactivating PTSD symptoms before therapy and that there was no fear response after EMDR.

The precuneus was the sole brain structure with increased metabolism after symptom remission. This increased metabolism was correlated with symptom evolution as measured by the CAPS. The precuneus metabolism measured before therapy also predicted the clinical evolution after EMDR therapy.

The precuneus is implied in autobiographical memory, multisensory integration and futureoriented thinking (2). In a recent meta-analysis of non-VRE studies, it appears to be a key structure that regulates the fear network in fear conditioning and extinction protocol (11). Due to its functions, we can assume that the precuneus can modulate the subjective experience of anxious and fearful states to reprocess the traumatic memory and discharge it from its emotional load (11). The memory is then perceived as a scene in which the patient is no longer involved instead of repeatedly reliving the memory. In other words, the subject becomes a neutral observer of the traumatic scene.

The main limitation of this study is the low number of participants ( $\mathrm{n}=15)$. Further experiments should reproduce our results with a wait-list group and go further by exploring the precise functions of the precuneus during EMDR therapy not only before and after therapy but also during the EMDR sessions.

In conclusion, this study shows that precuneus metabolic improvement while reliving war combat correlates with a reduction in PTSD symptoms after EMDR therapy. Moreover, precuneus metabolism before therapy was a predictor of symptom evolution. Our results could suggest therapeutic implications leading to the use of rTMS or neurofeedback for modulating precuneus activity. 


\section{Compliance with Ethical Standards:}

Funding: This work was conducted in the framework of DHU-Imaging thanks to the support of the A*MIDEX project ( ${ }^{\circ}$ ANR-11-IDEX-0001-02) ("Investissements d'Avenir » French Government programme, managed by the French National Research Agency (ANR)).

Conflicts of Interest: None

Ethics: Subjects gave written informed consent for their participation in accordance with the Declaration of Helsinki. The study was approved by the Institutional Review Board CPP Sud Méditerrannée (Ref: 2014-002126-12). 
Figure 1. Screen captures of the virtual environment created for the ${ }^{18} \mathrm{~F}-\mathrm{FDG}$ PET scan sensitized by virtual exposure consisting of an attack on their group by insurgents during a patrol in Afghanistan with several wounded. 
Table 1: Socio-demographics and clinical characteristics of the military patients 
Figure 2: After EMDR, in comparison to the baseline PET performed before EMDR, a significant increase in precuneus metabolism was exhibited $(\mathrm{p}<0.005$ uncorrected, $\mathrm{k}>180)$ during virtual reality exposure. After EMDR therapy, patients demonstrated increased metabolism in the precuneus prevailing on the right side. 
Figure 3: Significant correlation between PET metabolism of the precuneus and changes in clinical scale scores (PCLS T1-T0 and CAPS T1-T0).

The red correlation shows that the higher the metabolic value of the precuneus increased after EMDR, the symptoms were more reduced. The correlation in black shows that the higher the PET metabolism of the precuneus before EMDR, the more the PCLS scores decreased at the end of the therapy.

Precuneus T1-T0 vs CAPS T1-T0 $(r=-0.73$ and $p<0.001)$

Precuneus at T0 vs PCLS T1-T0 $(r=-0.667$ and $p<0.006)$

CAPS: Clinician Administered PTSD Scale

PCLS: Posttraumatic Stress Disorder Checklist Scale 


\section{References}

1. Xue C, Ge Y, Tang B, Liu Y, Kang P, Wang M, et al. A meta-analysis of risk factors for combat-related PTSD among military personnel and veterans. PloS One.

2015;10(3):e0120270.

2. Yehuda R, Hoge CW, McFarlane AC, Vermetten E, Lanius RA, Nievergelt CM, et al. Post-traumatic stress disorder. Nat Rev Dis Primer. 08 2015;1:15057.

3. Rousseau PF, El Khoury-Malhame M, Reynaud E, Zendjidjian X, Samuelian JC, Khalfa S. Neurobiological correlates of EMDR therapy effect in PTSD. Eur J Trauma Dissociation [Internet]. 30 juill 2018 [cité 8 août 2018]; Disponible sur: http://www.sciencedirect.com/science/article/pii/S2468749918300218

4. Pagani M, Di Lorenzo G, Verardo AR, Nicolais G, Monaco L, Lauretti G, et al. Neurobiological correlates of EMDR monitoring - an EEG study. PloS One. 2012;7(9):e45753.

5. Verger A, Guedj E. The renaissance of functional 18F-FDG PET brain activation imaging. Eur J Nucl Med Mol Imaging. 29 sept 2018;

6. Chiaravalloti A, Pagani M, Micarelli A, Di Pietro B, Genovesi G, Alessandrini M, et al. Cortical activity during olfactory stimulation in multiple chemical sensitivity: a (18)FFDG PET/CT study. Eur J Nucl Med Mol Imaging. avr 2015;42(5):733 - 40.

7. Verger A, Malbos E, Reynaud E, Mallet P, Mestre D, Pergandi J-M, et al. Brain metabolism and related connectivity in patients with acrophobia treated by virtual reality therapy: an 18F-FDG PET pilot study sensitized by virtual exposure. EJNMMI Res. 1 oct 2018;8(1):93.

8. American Psychiatric Association. Diagnostic and statistical manual of mental disorders. Text revision. 4th Edition Washington, DC. American Psychiatric Association; 2000.

9. Ventureyra VAG, Yao S-N, Cottraux J, Note I, De Mey-Guillard C. The validation of the Posttraumatic Stress Disorder Checklist Scale in posttraumatic stress disorder and nonclinical subjects. Psychother Psychosom. févr 2002;71(1):47- 53.

10. Blake DD, Weathers FW, Nagy LM, Kaloupek DG, Gusman FD, Charney DS, et al. The development of a Clinician-Administered PTSD Scale. J Trauma Stress. janv 1995;8(1):75- 90 .

11. Fullana MA, Albajes-Eizagirre A, Soriano-Mas C, Vervliet B, Cardoner N, Benet O, et al. Fear extinction in the human brain: A meta-analysis of fMRI studies in healthy participants. Neurosci Biobehav Rev. mai 2018;88:16- 25. 
Table 1: Socio-demographics and clinical characteristics of the military patients

$$
\text { EMDR Group }(\mathbf{n}=15)
$$

\section{Socio-demographic characteristic}

Age (years \pm SD)

Duration of illness (years $\pm S D$ )

Duration of EMDR therapy, number of sessions (sessions $\pm \mathrm{SD}$ )

Mean delay between the two FDG-PET (months)

\section{Use of psychiatric medications}

Selective serotonin reuptake inhibitors

\section{Psychometric scales}

Subjective unit of discomfort (mean \pm $\mathrm{SD})$

Posttraumatic Stress Checklist Scale (mean $\pm \mathrm{SD}$ )

Clinician-Administered PTSD Scale (mean $\pm \mathrm{SD}$ )

$$
\begin{gathered}
36.8 \pm 8.88 \\
5.8 \pm 0.75 \\
4.46 \pm 0.54 \\
3.05 \pm 0.78
\end{gathered}
$$

$$
\mathrm{n}=5
$$

TO

T1

$\begin{array}{crr}6 \pm 2.26 & 1.2 \pm 1.42 & 0.001 \\ 62.13 \pm 8.18 & 24.4 \pm 8.05 & 0.001 \\ 78.06 \pm 12.25 & 13.86 \pm 12.68 & 0.001\end{array}$

$$
\begin{gathered}
n=9 \\
n=2 \\
n=12 \\
n=1
\end{gathered}
$$$$
\mathrm{n}=0
$$$$
\mathrm{n}=0
$$$$
0.005
$$$$
\mathrm{n}=2
$$$$
>0.05
$$$$
\text { Agoraphobia }
$$$$
\mathrm{n}=0
$$$$
0.005
$$$$
>0.05
$$ 

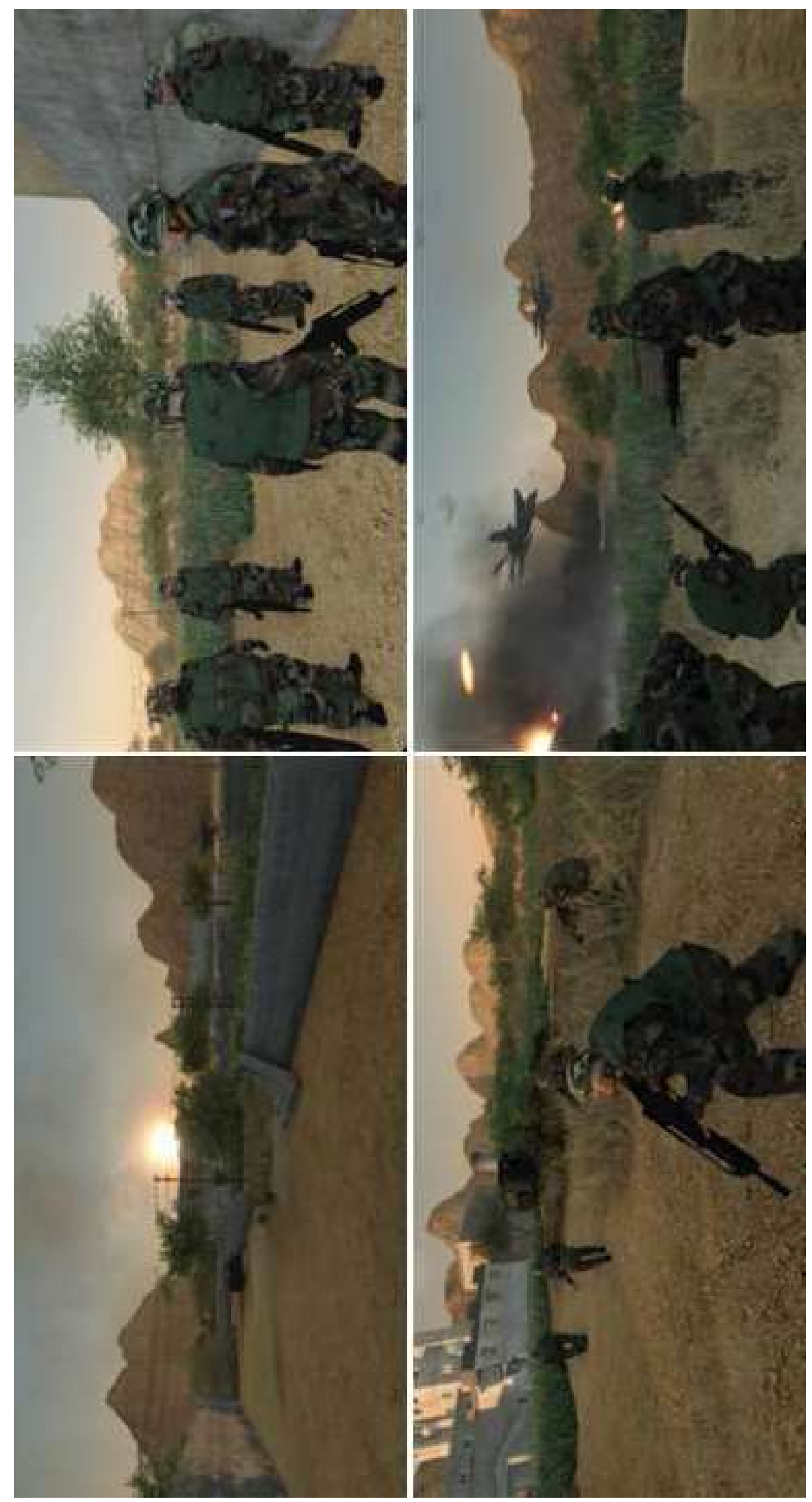

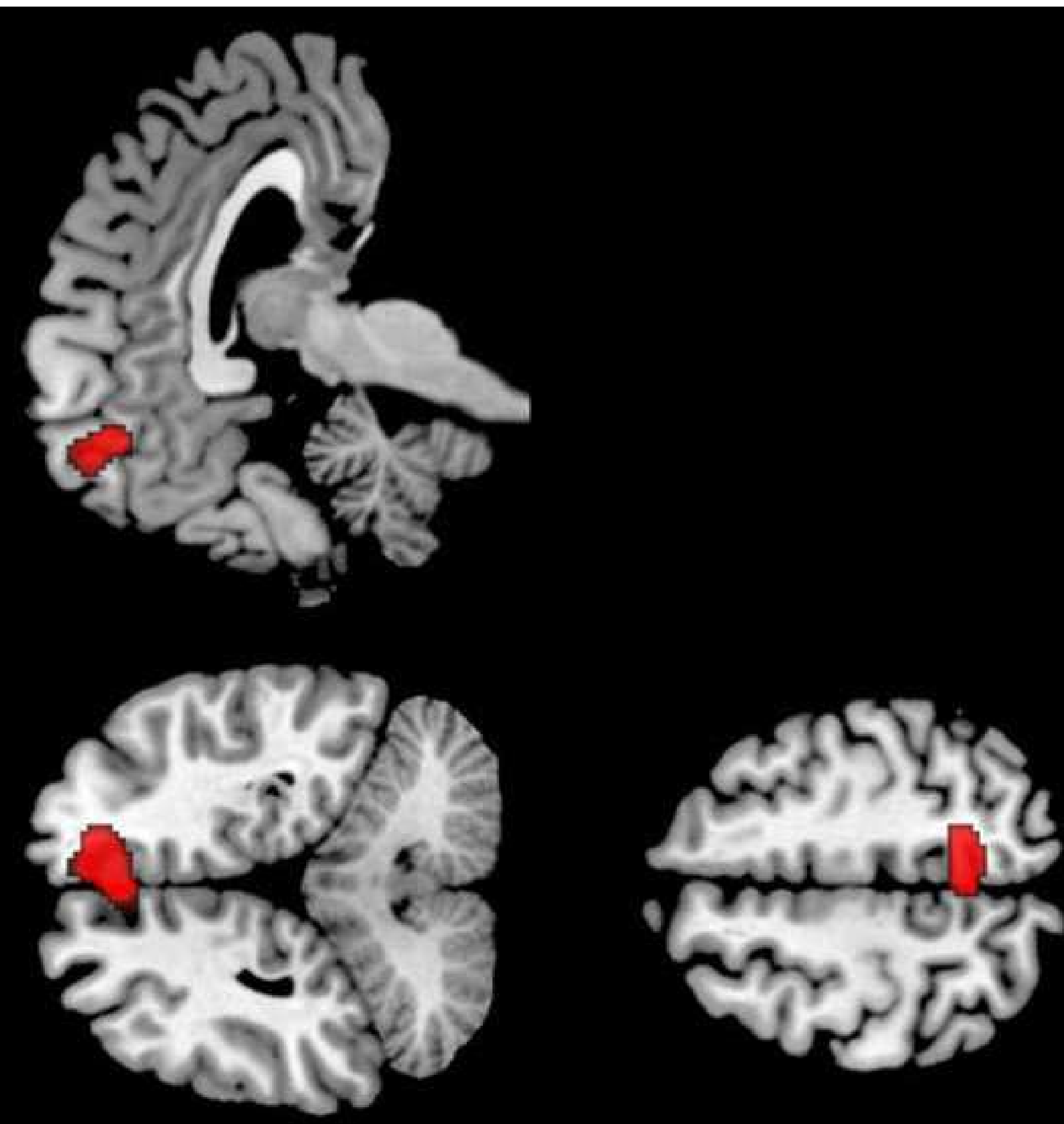


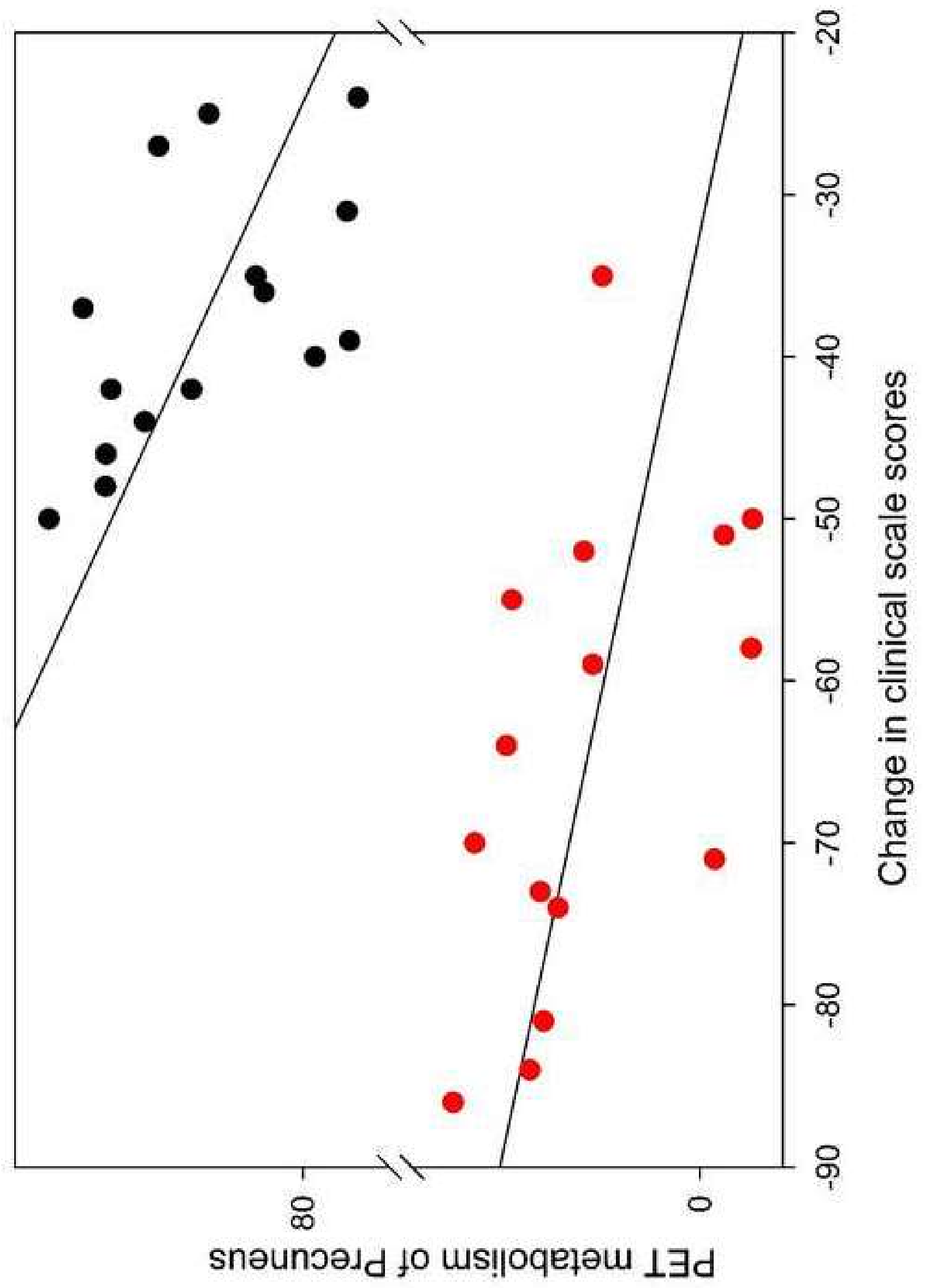

$m$
$\frac{\mathbb{d}}{丂}$
$\frac{\sqrt{2}}{4}$ 